\title{
Live Cell Imaging Reveals the Relocation of dsRNA Binding Proteins Upon Viral Infection
}

\author{
Deborah A. Barton, ${ }^{1}$ Elke F. Roovers, ${ }^{1,2}$ Quentin Gouil,, ${ }^{1,3}$ Guilherme C. da Fonseca, ${ }^{1,4}$ Rodrigo S. Reis, ${ }^{1}$ \\ Craig Jackson, ${ }^{1}$ Robyn L. Overall, ${ }^{1}$ Adriana F. Fusaro, ${ }^{1}$ and Peter M. Waterhouse ${ }^{1,5}$ \\ ${ }^{1}$ University of Sydney, Sydney, NSW, 2006, Australia; ${ }^{2}$ Institute of Molecular Biology, Mainz, Germany; ${ }^{3}$ Centre for \\ AgriBioscience, Department of Animal, Plant and Soil Sciences, School of Life Sciences, La Trobe University, Victoria 3086; \\ ${ }^{4}$ Universidade Federal do Rio Grande do Sul, RS, Brazil; and ${ }^{5}$ Queensland University of Technology, Brisbane, QLD, Australia
}

Accepted 10 March 2017.

\begin{abstract}
Viral infection triggers a range of plant responses such as the activation of the RNA interference (RNAi) pathway. The double-stranded RNA binding (DRB) proteins DRB3 and DRB4 are part of this pathway and aid in defending against DNA and RNA viruses, respectively. Using live cell imaging, we show that DRB2, DRB3, and DRB5 relocate from their uniform cytoplasmic distribution to concentrated accumulation in nascent viral replication complexes (VRC) that develop following cell invasion by viral RNA. Inactivation of the DRB3 gene in Arabidopsis by T-DNA insertion rendered these plants less able to repress RNA viral replication. We propose a model for the early stages of virus defense in which DRB2, DRB3, and DRB5 are invasion sensors that relocate to nascent VRC, where they bind to viral RNA and inhibit virus replication.
\end{abstract}

Many different virus- and host-encoded proteins are involved in the battle that ensues when a virus infects a cell (Ding 2010; Mandadi and Scholthof 2013). Most often, in plants, the invading virus has an RNA genome and the host's antiviral defense relies on a mechanism called RNA interference (RNAi) or RNA silencing. RNAi pathways are omnipresent in eukaryotes; have a number of common protein components, including Dicers, Argonautes (AGO), and double-stranded (ds)RNA-binding (DRB) proteins; and are guided by small (s)RNA (Eamens et al. 2008). In the model plant Arabidopsis thaliana, there are four Dicer-like (DCL1 to 4), 10 AGO, and 5 DRB proteins (Curtin et al. 2008; Margis et al. 2006; Vaucheret 2008). Some of these appear to be exclusively involved with endogenous gene regulation, but many have roles in virus defense.

All four DCL cleave viral dsRNA into short interfering (si)RNA with differing efficiency; however, this processing is predominantly mediated by DCL4, aided by DRB4, and the siRNA are loaded onto AGO1, AGO2, AGO5, AGO7, and

Current address of R. S. Reis: University of Lausanne, Lausanne 1015, Switzerland.

Current address of A. F. Fusaro: Universidade Federal do Rio de Janeiro, Rio de Janeiro, RJ, Brazil.

Corresponding authors: D. Barton; Telephone: +61 29351 3285; Fax: +61 29351 4771; E-mail: deborah.barton@sydney.edu.au; and A. F. Fusaro; E-mail: adriana.fusaro1@gmail.com

*The $\boldsymbol{e}$-Xtra logo stands for "electronic extra" and indicates that one supplementary figure and one supplementary table are published online.

@ 2017 The American Phytopathological Society
AGO10 (Deleris et al. 2006; Fusaro et al. 2006; Garcia-Ruiz et al. 2015). Guided by siRNA, the AGO recognize and cleave invading single-stranded viral RNA and also trigger a secondary amplification response by RNA-dependent RNA polymerase 6 (RDR6) (Dalmay et al. 2000) in concert with other proteins such as suppressor of gene silencing 3 (SGS3) (Mourrain et al. 2000). This description of the pathway is mainly based on interpreting the mechanistic actions of the DCL, AGO, DRB4, RDR6, and SGS3 by analysis of virus-challenged Arabidopsis mutant lines (Ding and Voinnet 2007; Llave 2010). Recently, using a similar mutant-based approach, DRB3 has also been shown to play a role in viral defense, through aiding the methylation of invading DNA viruses (Raja et al. 2014). However, the functions of the other members of the DRB are unclear and the intracellular locations of these proteins, especially during viral invasion, have not been closely examined. Curiously, all four DCL and DRB4 appear to be completely or predominantly restricted to the nucleus, despite RNA viruses having entirely cytoplasmic life cycles (Hoffer et al. 2011; Jakubiec et al. 2012; Schoelz et al. 2011) and this has raised questions about how and where the RNAi system interfaces with its target viruses (Llave 2010; Waterhouse and Fusaro 2006).

Until recently, models of viral replication and movement within a plant cell have been based on animal virus models and considered to be spatially separated processes (Lucas 2006; Schoelz et al. 2011; Verchot-Lubicz et al. 2010). However, work (Tilsner et al. 2013) examining Potato virus $X$ (PVX) provides new insights and a model for viral trafficking that includes coreplicational insertion of viral RNA into the plasmodesmata (PD), the intercellular channels that connect plant cells. In order to better understand the processes involving DRB that occur within an infected plant, we fluorescently tagged the five DRB proteins from Arabidopsis and their orthologs from Nicotiana benthamiana, and examined their behaviors in response to six viruses in Arabidopsis or $N$. benthamiana plants.

Some plant viruses replicate in the cell without causing visible cytopathology, except for the production of virus particles, whereas others have obvious viral replication complexes (VRC) (Schoelz et al. 2011). To more easily recognize the locations of DRB-virus responses, we studied PVX, Tobacco mosaic virus (TMV), Turnip mosaic virus (TuMV), Tomato spotted wilt virus (TSWV), Turnip yellow mosaic virus (TYMV), and Cucumber mosaic virus (CMV), which all, with the exception of CMV, induce visible VRC. These viruses were also selected because each is a member of a different virus family (Alphaflexiviridae, Virgaviridae, Potyviridae, Bunyaviridae, Tymoviridae, and Bromoviridae) (King et al. 2011) and infect either Arabidopsis or N. benthamiana or both. 

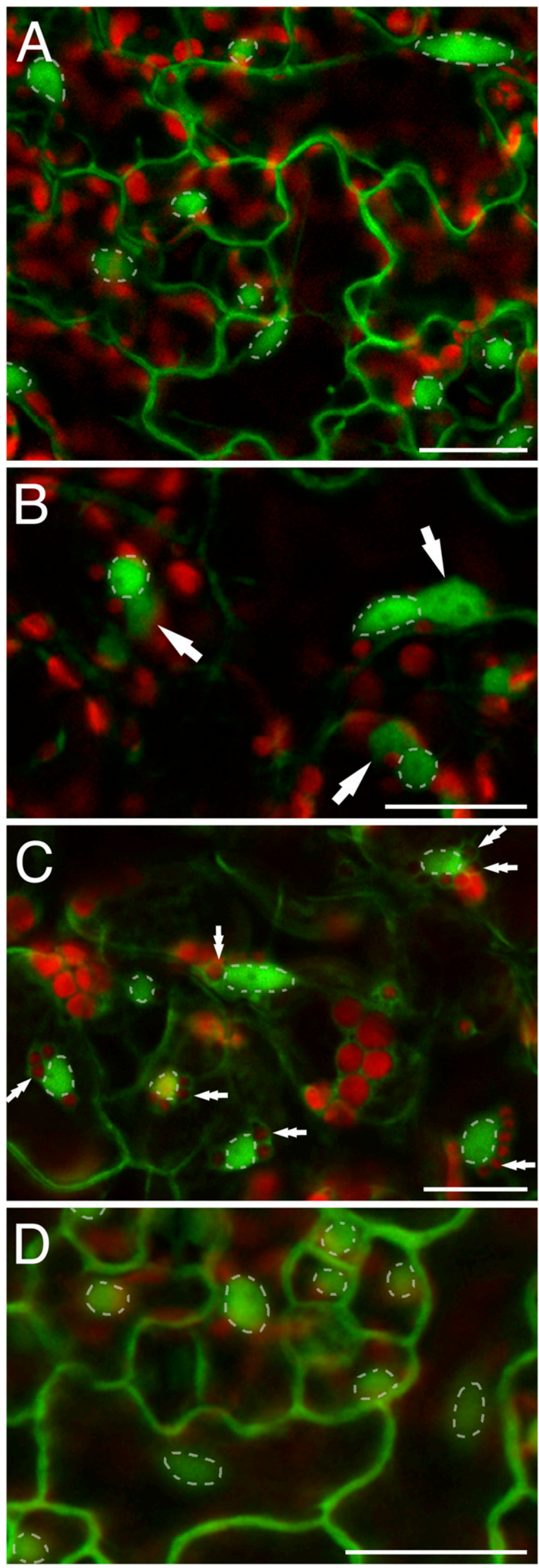

\section{RESULTS}

Arabidopsis plants stably expressing the green fluorescent protein (GFP) diffusely within the cytoplasm (cytoplasmic GFP) were inoculated with CMV, TSWV, TuMV, or TYMV and their leaf epidermal cells were examined 5 to 14 days later to determine whether there were obvious cytological signs of infection. In uninfected cells, GFP was located in the cortical cytoplasm at the boundaries of the epidermal pavement cells as well as within transvacuolar strands and in the nuclei (Fig. 1A). In all the images presented in this article, care has been taken to identify the nucleoli wherever possible in order to verify the identification of the nuclei. Cells infected with TSWV (Fig. 1B) or TuMV typically contained a large, dense, cytoplasmic mass - a VRC — adjacent to nuclei. There was less cytoplasm at the cellular peripheries and the majority of the cells' cytoplasm accumulated into the VRC after infection. TYMV infection induced the aggregation of chloroplasts or plastids around the nuclei; these appeared as dark, round bodies surrounded by the fluorescent GFP (Fig. 1C). There was no visible sign of CMV infection within the cells (Fig. 1D), although the tissue selected for examination was from chlorotic regions of leaves with obvious virus symptoms.

To investigate which DRB interact with the viruses, Arabidopsis plants were stably transformed with constructs designed to express fluorescently tagged fusions of these proteins (Fig. 2). Tagged DRB1 and DRB4 were confined to the nucleus, with DRB4 forming one, sometimes two, bright fluorescent speckles near the boundary of the nucleolus. In contrast, fluorescently tagged DRB2, DRB3, and DRB5 were visible in both the cytoplasm and nucleus. The plants were then challenged with CMV, TSWV, TuMV, or TYMV. Infected cells expressing the fluorescent DRB were compared with infected cells expressing cytoplasmic GFP. DRB1 and DRB4 remained in the nuclei of all infected cells. By contrast, in TuMV- or TSWV-infected cells, DRB2, DRB3, and DRB5 invariably entered the VRC and formed small, bright punctae within them. These punctae were absent from infected cells expressing cytoplasmic GFP, suggesting a possible interaction between DRB2, DRB3, and DRB5 and a structure associated with the viruses. DRB2 also formed bright speckles in the area between the nucleus and aggregated chloroplasts in TYMV-infected cells and in nuclei of CMV-infected cells. DRB3 and DRB5 were in the cytoplasm around chloroplasts but did not form bright speckles in TYMV-infected cells.

Until now, DRB2, DRB3, and DRB5 have not been associated with antiviral responses to RNA viruses, although DRB3 has a role in defense against DNA viruses by aiding methylation (Raja et al. 2014). To investigate any involvement of DRB2, DRB3, and DRB5 in more detail and to make use of the wellcharacterized viruses TMV and PVX (which do not infect Arabidopsis), we altered our approach to using transient transgene expression in $N$. benthamiana. AtDRB-orange fluorescent fusion proteins were transiently expressed in $N$. benthamiana leaves. The intracellular locations of these fluorescent fusion proteins in the leaf epidermal cells of $N$. benthamiana were almost identical to those in Arabidopsis, with the exception of DRB1, which was

Fig. 1. Virus infection of Arabidopsis leaf epidermal cells expressing cytoplasmic green fluorescent protein (GFP). Dashed lines surround all nuclei. A, In uninfected cells, GFP is diffuse throughout the cytoplasm and nuclei. B, In cells infected with Tomato spotted wilt virus, viral replication complexes (arrows) form at one side of nuclei. C, In Turnip yellow mosaic virus-infected epidermal cells, chlorophyll containing plastids (red) cluster around nuclei and are surrounded by GFP (double arrows). D, Cells infected with Cucumber mosaic virus are similar in appearance to uninfected cells. Scale bars: $20 \mu \mathrm{m}$. 
concentrated in the nucleolus as well as being present in the nucleus (Fig. 3). DRB4 appeared almost exclusively located in the nuclei, whereas DRB2, DRB3, and DRB5 were present in both the cytoplasm and nuclei. As observed in Arabidopsis (Fig. 2), there was no obvious change in the location of the DRB following infection by CMV. When challenged with TuMV and TMV (using a strain of TMV that encodes GFP in its genome), DRB1 and DRB4 remained in nuclei whereas DRB2, DRB3, and DRB5 invariably entered VRC and formed bright concentrations within these. Again, these concentrations were absent from the VRC in cells expressing cytoplasmic orange fluorescent protein (OFP), suggesting that the fluorescent DRB fusions used in this study are translocated.

The locations and interactions of viral RNA and virus-encoded proteins in the VRC of PVX-infected $N$. benthamiana have been described in great detail (Linnik et al. 2013; Tilsner et al. 2012, 2013). We took advantage of this knowledge to provide context for our DRB studies. To monitor the virus-DRB interactions, an infectious PVX-overcoat construct (Cruz et al. 1996) was modified to encode cyan fluorescent protein (CFP) fused to the viral coat protein $(\mathrm{CP})$. This was agroinfiltrated into $N$. benthamiana leaves in combination with the tagged AtDRB expression constructs. DRB1 and DRB4 accumulated indistinguishably in the nuclei of infected and uninfected cells and could not be seen in VRC (Fig. 3). During PVX infection, DRB2, DRB3, and DRB5 accumulated to high levels in VRC and invariably relocated from nuclei and the cytoplasm into VRC. Intriguingly, DRB2, DRB3, and DRB5 appeared as ring-like structures in the VRC. Repeating this analysis using DRB proteins from $N$. benthamiana gave essentially the same response (Fig. 4).

The interactions of PVX-encoded proteins and genomic RNA have been elucidated within VRC and at PD by high- and superresolution imaging (Linnik et al. 2013; Tilsner et al. 2009, 2013). Among the many interactions, triple-gene block 1 (TGB1), a PVX-encoded movement protein, was shown to be both a

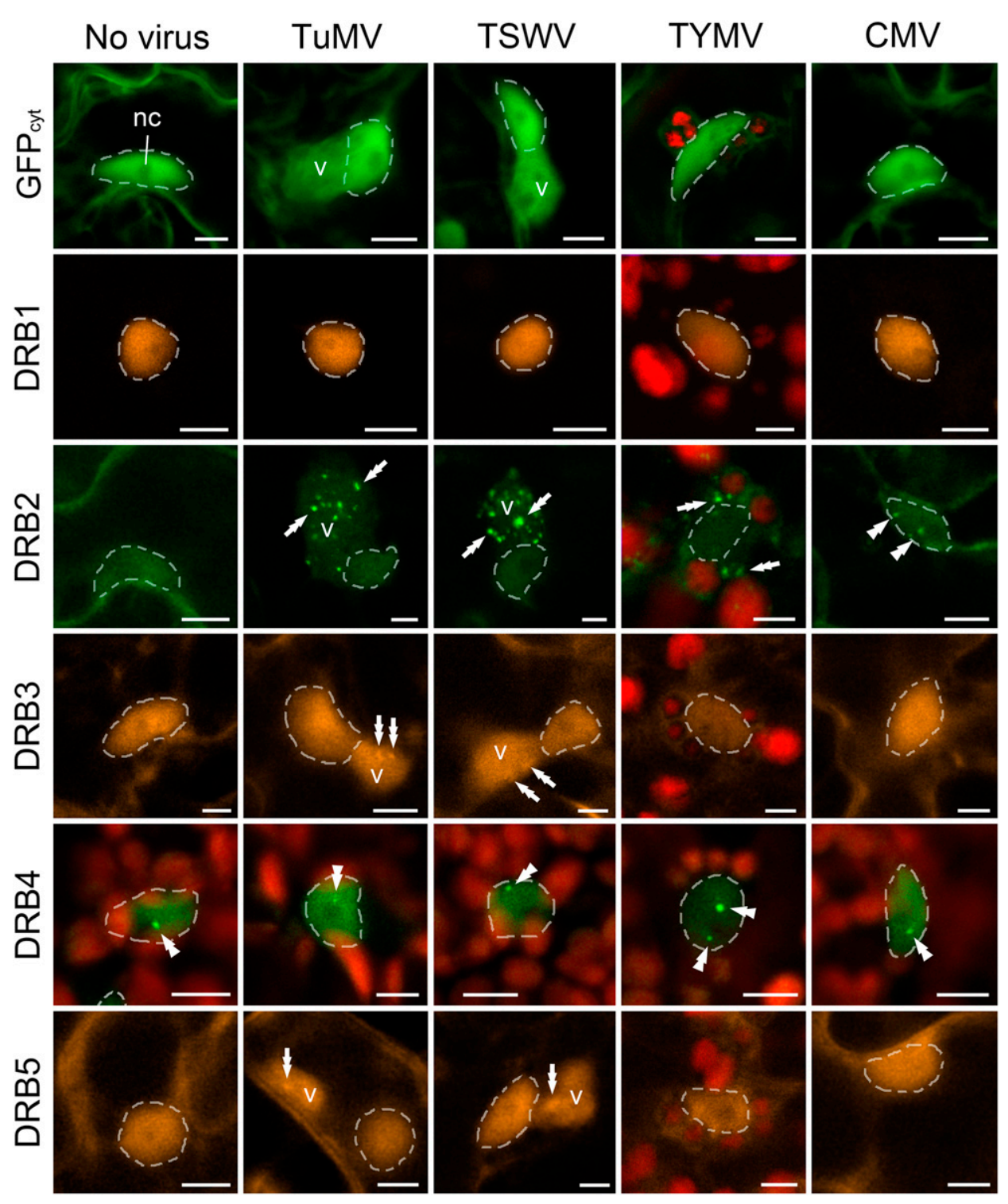

Fig. 2. Double-stranded RNA binding (DRB)2, DRB3, and DRB5 form discrete concentrations in viral replication complexes (VRC) of infected Arabidopsis cells. Uninfected and infected Arabidopsis leaf epidermal cells constitutively expressing fluorescent fusions DRB1-orange fluorescent protein (OFP), DRB2green fluorescent protein (GFP), DRB3-OFP, DRB4-GFP, DRB5-OFP, or cytoplasmic GFP (control). VRC are marked by 'v'; double arrows point to bright, fluorescent bodies in VRC; double arrowheads point to fluorescent bodies in nuclei. Autofluorescent plastids (red) are included for Turnip yellow mosaic virus (TYMV)-infected cells and DRB4 images. In TYMV-infected cells, plastids are clustered around nuclei and are surrounded by a ring of cytoplasm. In all panels, nuclei are surrounded by dashed lines and, wherever possible, the nucleolus (nc) was focused on to aid in the identification of nuclei. TuMV $=$ Turnip mosaic virus, TSWV = Tomato spotted wilt virus, and CMV = Cucumber mosaic virus. Scale bars: $5 \mu \mathrm{m}$. 
scaffold surrounded by viral RNA in the VRC (Tilsner et al. 2012) and an essential component for trafficking viral RNA through PD (Tilsner et al. 2013). Therefore, we made a TGB1yellow fluorescent protein (YFP) construct and used it as a marker to better observe the locations and interactions of the
DRB within VRC. In these experiments, we examined cells simultaneously expressing TGB1-YFP and OFP-labeled DRB2, DRB3, or DRB5 within $N$. benthamiana leaves infected with PVX producing CFP-labeled CP (Fig. 5). This recapitulated but in greater detail the results shown in Figures 3 and 4, with each

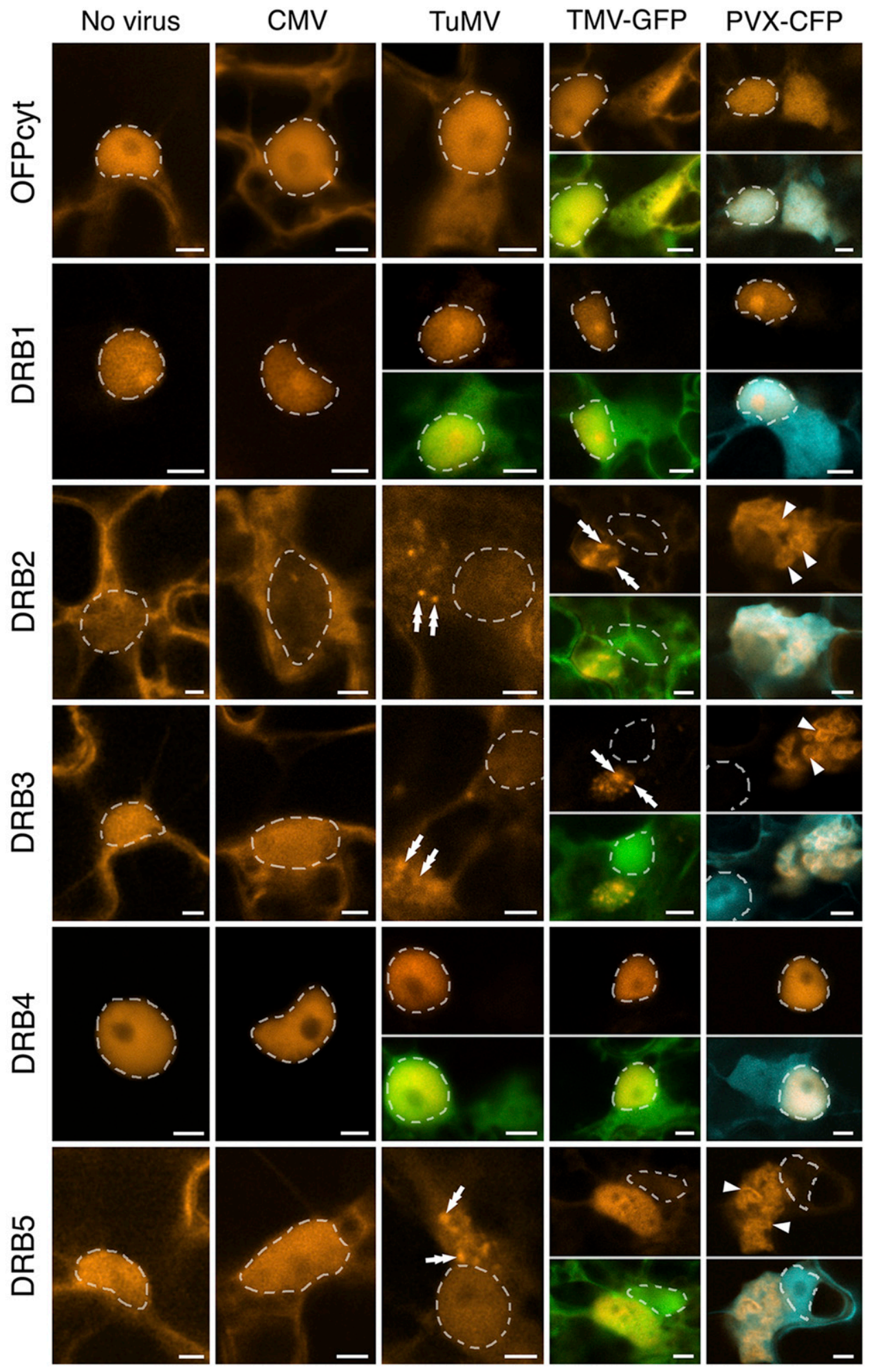

Fig. 3. Location of AtDRB1 to AtDRB5 during virus infection in Nicotiana benthamiana. Double-stranded RNA binding (DRB)2, DRB3, and DRB5 form bright structures in viral replication complexes (VRC). Transient expression of DRB1-orange fluorescent protein (OFP), DRB2-OFP, DRB3-OFP, DRB4-OFP, DRB5-OFP and cytoplasmic OFP in $N$. benthamiana leaf epidermal cells. Cells were infected with Cucumber mosaic virus (CMV), Turnip mosaic virus (TuMV), Tobacco mosaic virus (TMV)-green fluorescent protein (GFP), or Potato virus X (PVX)-cyan fluorescent protein (CFP) (blue). Cytoplasmic GFP (GFPcyt) was coexpressed with DRB1-OFP or DRB4-OFP in TuMV-infected tissue to show the position of VRC. The GFP in TMV-GFP images was produced during infection as part of the TMV genome. Double arrows point to bright spots in TuMV and TMV VRC and arrowheads point to ring-like arrangements in PVX VRC. Nuclei are surrounded by dashed lines. Scale bars: $5 \mu \mathrm{m}$. 


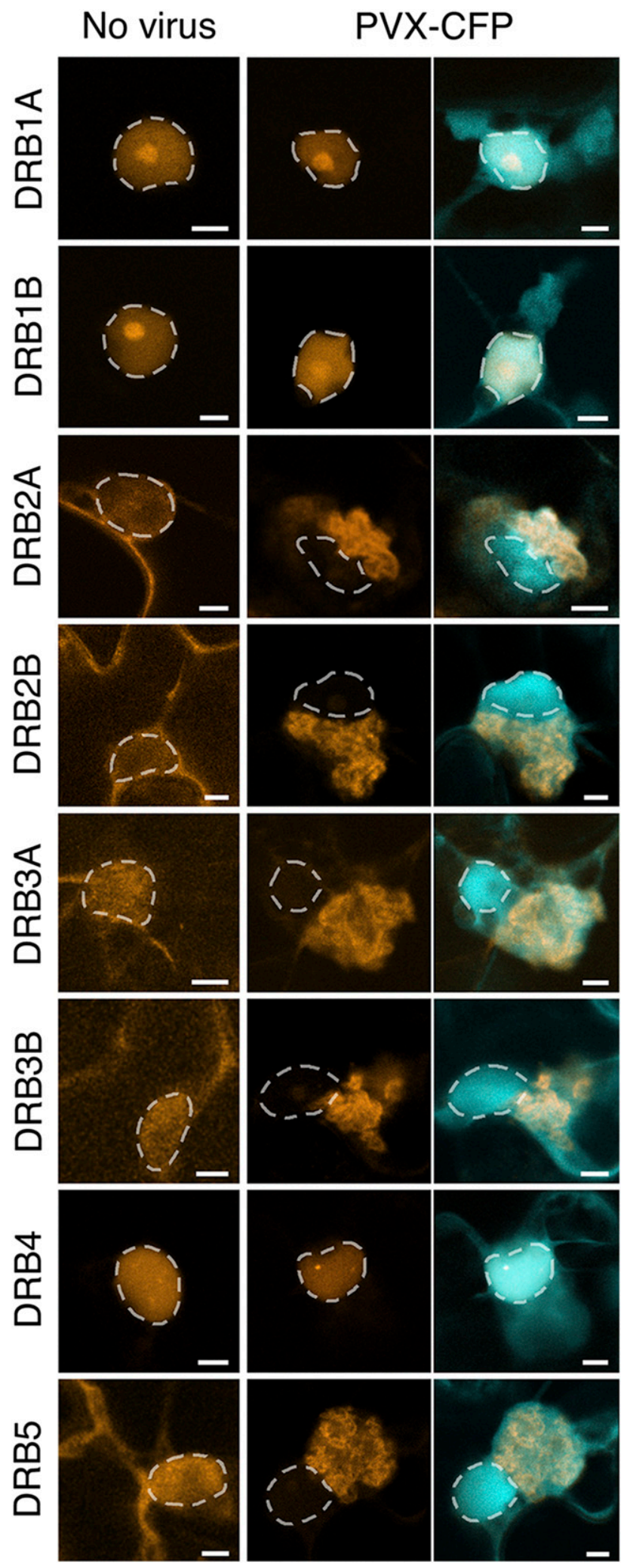

Fig. 4. Nicotiana benthamiana double-stranded RNA binding (DRB)2A, DRB2B, DRB3A, DRB3B, and DRB5 also form ring-like structures in Potato virus $X$ (PVX) viral replication complexes (VRC). Location of $N$. benthamiana DRB fused to orange fluorescent protein and transiently expressed in N. benthamiana leaves, with or without coexpression of PVXcyan fluorescent protein (CFP). DRB1A, DRB1B, and DRB4 remain in nuclei of infected cells. DRB2A, DRB2B, DRB3A, DRB3B, and DRB5 are in the cytoplasm and nuclei of uninfected cells and form bright, fluorescent ring-like structures in the VRC of PVX-infected cells. Nuclei are surrounded by dashed lines. Scale bars: $5 \mu \mathrm{m}$. of the DRB forming ring-like structures within the VRC. Additionally, it revealed that the centers of the rings were occupied by TGB1. Comparing these images with those of Tilsner et al. (2009) shows that the locations of the DRB map to those of PVX RNA, around TGB1 foci. This insight, coupled with identification of dsRNA binding sites in all three DRB and the demonstration by gel shift assay that DRB2 binds to dsRNA but not single-stranded RNA (Hiraguri et al. 2005), strongly suggests that DRB2, DRB3, and DRB5 are specifically binding to PVX RNA, and probably the double-stranded form. This is also consistent with the demonstration that viral RNA-dependent RNA polymerase and dsRNA are located in vesicles found in TuMVinfected cells (Cotton et al. 2009). Tagged DRB1 and DRB4 were included in the experiment and, in the rare cells where one of these was massively overexpressed, some of the protein entered the cytoplasm and, in infected cells, penetrated into the VRC (Supplementary Fig. S1). However, neither DRB1 nor DRB4 formed ring-like structures around TGB1, suggesting that they were not able to preferentially bind to viral RNA. Although this is consistent with the well-known attribute of DRB1 that only recognizes imperfectly duplexed regions of primary micro (mi)RNA transcripts, it was surprising to observe this for DRB4, which might have been expected to bind to its presumed template.

The promoters of $D R B 2, D R B 3$, and $D R B 5$ driving reporter genes in Arabidopsis have shown that DRB3 is expressed throughout the plant, especially in the vasculature, whereas expression patterns of $D R B 2$ and $D R B 5$ are largely restricted to the apical meristem (Curtin et al. 2008). From this it seemed that, if these proteins were involved in viral defense, DRB3 would play the predominant role. Therefore, we examined tissues expressing OFP-labeled DRB3 (Fig. 6) at the invasion front of a PVX-CFP infection in $N$. benthamiana. This revealed cells at different stages of virus invasion and provided insights into the early processes. Cells at very early stages of infection could be seen adjacent to well-infected cells. In the uninfected or newly

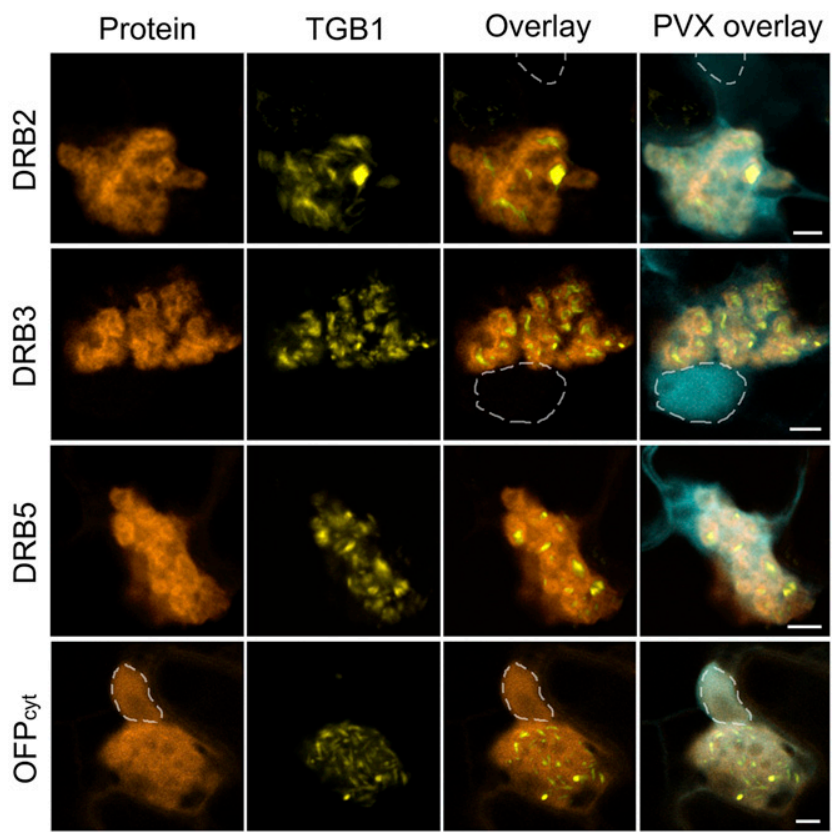

Fig. 5. Double-stranded RNA binding (DRB)2, DRB3, and DRB5 form ringlike structures around triple-gene block 1 (TGB1) within Potato virus $X$ (PVX) viral replication complexes (VRC). Coexpression of DRB2-orange fluorescent protein (OFP), DRB3-OFP, DRB5-OFP, or cytoplasmic OFP (OFPcyt) (orange) with TGB1-yellow fluorescent protein (yellow) in PVX-cyan fluorescent protein (blue)-infected Nicotiana benthamiana leaf epidermal cells. DRB2, DRB3, and DRB5 form ring-like structures around TGB1 in VRC whereas cytoplasmic OFP remains diffuse. Nuclei are surrounded by dashed lines. Scale bars: $5 \mu \mathrm{m}$. 
invaded cells, OFP-labeled DRB3 was widely distributed in the cytoplasm, highlighting the outline of the cell in orange, whereas well-infected cells were outlined in blue by CFP expressed from the virus (Fig. 6A and B). TGB1 has been shown localizing to PD to facilitate transport of viral RNA into adjacent cells (Tilsner et al. 2013). By incorporating TGB1-YFP into our assay, the very earliest stages of infection were captured. DRB3-OFP intensified adjacent to the TGB1-YFP foci (Fig. 6C and D) and increasingly large structures containing DRB3-OFP were observed in different cells, with their sizes correlating directly with increasing CFP and decreasing OFP distribution in the cytoplasm (Fig. 6E to H). These VRC increased in size the closer they were to the nucleus, and DRB3-OFP concomitantly resolved into ring-like structures similar to those observed in the perinuclear VRC. This series of images strongly suggests an ordered timeline of events: first, the invading viral RNA is recognized by DRB3 soon after entering the cell through the PD; then, DRB3 increasingly relocates to the site of this interaction from elsewhere in the cytoplasm and nucleus. As the DRB3 relocates, the VRC continues to enlarge and migrate toward the nucleus.

The association of DRB3 with viral RNA and the formation and movement of the VRC suggested to us either that the protein might be playing a role in repressing the virus or that DRB3 is used by the virus to camouflage itself from the cell's defenses. To address this question, wild-type Arabidopsis plants and those of a transgenic line containing a T-DNA insertion in DRB3 (drb3) and a similar line with an insertion in DRB4 (drb4) were inoculated with TuMV, and the virus titers were measured by enzyme-linked immunosorbent assay (ELISA) 3 weeks later. The virus accumulated to significantly higher levels in $d r b 4$ than in wild-type plants, as expected (Curtin et al. 2008; Qu et al. 2008), and also accumulated to higher levels in drb3 (Fig. 7). This shows that both DRB3 and DRB4 have roles in repressing viral replication.

\section{DISCUSSION}

Some of the earliest experiments that revealed the existence of the RNAi pathway and provided information about its components and mechanism have come from studying viruses in plants (Ding and Voinnet 2007; Lindbo and Dougherty 1992; Hamilton and Baulcombe 1999; Mlotshwa et al. 2008; Voinnet et al. 1999; Wang et al. 2000; Waterhouse et al. 1998). DCL4, DCL2, and DCL3 produce high levels of 21-, 22-, and 24nucleotide (nt) siRNA from the RNA of invading viruses (Blevins et al. 2006; Fusaro et al. 2006; Llave 2010) and these guide AGO
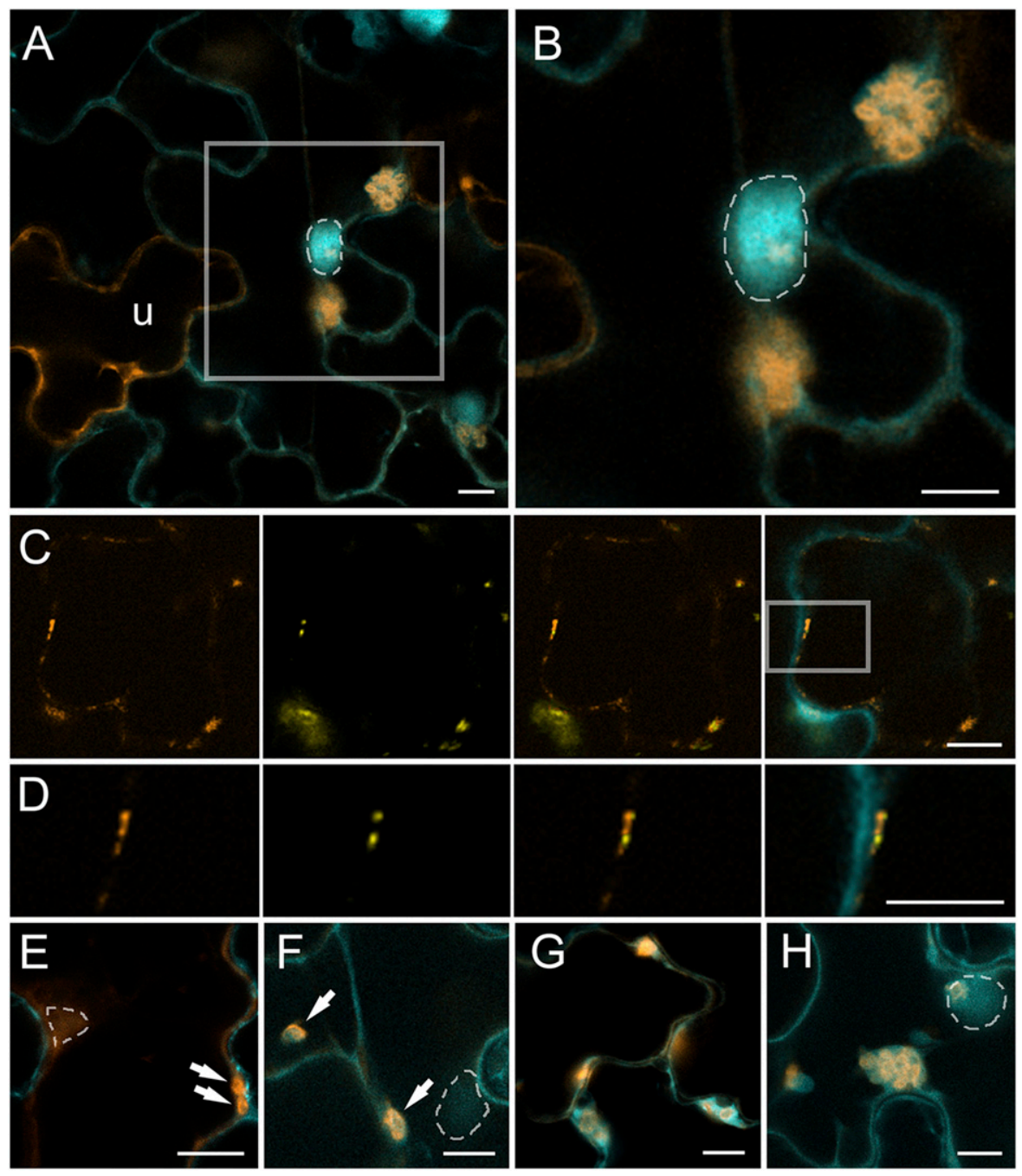

Fig. 6. Double-stranded RNA binding (DRB)3 localization during stages of Potato virus $X$ (PVX) infection and viral replication complex (VRC) formation Location of DRB3-orange fluorescent protein (OFP) (orange) and triple-gene block 1 (TGB1)-yellow fluorescent protein (yellow) during PVX-cyan fluorescent protein (CFP) (blue) infection of Nicotiana benthamiana leaf epidermal cells. A, DRB3 is in the cytoplasm of an uninfected cell (u) whereas it concentrates in bright rings within two VRC of an infected cell. B, The boxed region in A, at a higher zoom. C, Early infection of a cell. TGB1 forms spots in the cytoplasm and DRB3 concentrates at these sites. D, Higher zoom of the box in C, showing the DRB3 starting to concentrate around the TGB1 spots. E to H, Overlays of DRB3-OFP and PVX-CFP show incremental stages of PVX infection and VRC formation. Nuclei are surrounded by dashed lines. Scale bars: $10 \mu \mathrm{m}$. 
family members to cleave viral RNA or recruit cellular machinery to methylate viral DNA. The roles and actions of DRB in viral defense are less clear. Of the five Arabidopsis DRB, only DRB4 appears to be involved in the production of viral siRNA (Curtin et al. 2008). It partners with DCL4 to cleave them from viral dsRNA (Curtin et al. 2008; Hiraguri et al. 2005). Curiously, DRB4 appears to be largely or exclusively restricted to the nucleus (Hiraguri et al. 2005; Pontes et al. 2013; this study), and recent work (Pumplin et al. 2016) suggests that DRB4 takes DCL4 into the nucleus to play a part in directing chromatin modification. The mechanism through which DRB4 interacts with DCL4 and cytoplasmic viral RNA and the location of this interaction remain unclear. DRB1 partners with DCL1 and aids it to cleave miRNA from precursor transcripts but rarely to produce siRNA from viral dsRNA. Although DRB2 and DRB5 have been reported to bind to DCL3 (Hiraguri et al. 2005), drb2, drb3, and drb5 mutants in Arabidopsis show no detectable reduction in the production of 24-, 21-, or 22-nt viral or transposon siRNA (Curtin et al. 2008; Raja et al. 2014). Nonetheless, DRB3 participates in methylation-mediated defense against geminiviruses (Raja et al. 2014) and DRB2 plays a role in determining whether an miRNA acts by translational repression (Reis et al. 2015).

We sought to gain greater insight into the involvement of the DRB in viral defense by live cell imaging of these proteins in uninfected and virus-infected Arabidopsis and $N$. benthamiana. This gave little evidence of DRB4 relocation into the cytoplasm of infected cells for any of the six viruses tested, including TYMV in Arabidopsis, unlike Jakubiec et al. (2012), who saw DRB4 relocation into the cytoplasm of TYMV-infected Arabidopsis protoplasts. However, a low level of DRB4 relocation into the PVX VRC in $N$. benthamiana could be detected if DRB4 was greatly overexpressed such that it had entered the cytoplasm of uninfected cells. In contrast, DRB2, DRB3, and DRB5 in both Arabidopsis and $N$. benthamiana substantially relocated to the VRC of four different viruses and formed bright punctae (TuMV, TSWV, and TMV) or rings (PVX) within them, which are likely to represent the sites of viral RNA. Closer examination of how these proteins behaved during PVX infection, coupled with the prior analysis of the roles and cellular locations of PVX-encoded proteins (Linnik et al. 2013; Schoelz et al. 2011; Tilsner et al. 2012, 2013; Verchot-Lubicz et al. 2010), provides an insight into the sequence of events in the virus-DRB interactions. DRB3 rapidly accumulated near the PD of newly virus-invaded cells. This may be indirectly visualizing the formation of the motile endoplasmic reticulum-associated granules, nascent VRC, and the virus starting to replicate (Tilsner et al. 2013). The defining feature of DRB is their dsRNA binding domains, suggesting that DRB3 may be binding to the nascent replicative form of the viral RNA. DRB1 and DRB4 have been shown to partner DCL1 and DCL4, respectively, to enhance their cleavage of dsRNA into mi- or siRNA. However, neither of two studies (Curtin et al. 2008; Raja et al. 2014) using $d r b 3$ was able to see a reduction in the production of DCL3-dependent 24-nt sRNA. With the demonstration that $d r b 3$ plants are more susceptible to both a DNA virus (Raja et al. 2014) and an RNA virus (this study), this suggests that DRB3 confers its antiviral action in some other way. Both DRB3 and DRB5 have been shown to be involved with translational repression (Eamens et al. 2012; Reis et al. 2015). Perhaps DRB3 is inhibiting viral RNA translation or replicase activity, restraining the virus, and allowing the cell's degradative RNAi mechanism more time to become fully functional and effective. Alternatively, DRB3 and, possibly, DRB2 and DRB5 may act at the early stages of viral defense as invasion sensors that relocate to nascent VRC, where they bind to viral RNA and help recruit the cell's antiviral machinery to inhibit virus replication.

The widespread expression of DRB3 in the vasculature and restriction of DRB2 and DRB5 to the apical tissues in Arabidopsis is intriguing. Perhaps the function of DRB2 and DRB5 is to provide increased protection to the floral meristem, thereby preventing viruses from invading reproductive tissues and being transmitted through the seed. Whatever the mechanisms involved, our results demonstrate that DRB2, DRB3, and DRB5 are among the first host-encoded proteins to engage with viral RNA entering a plant cell and that DRB3 is actively inhibiting RNA virus replication.

\section{MATERIALS AND METHODS}

\section{Constructs.}

The binary vectors 35S::PVX.CFP-CP and 35S::PVX.mCherry$\mathrm{CP}$, carrying modified cDNA of the $\mathrm{PVX}$ genome which give rise to viral $\mathrm{CP}$ fused with a fluorescent protein $(\mathrm{FP})$ via a $2 \mathrm{~A}$ peptide linker, were generated as follows. A 3.2-kb fragment of pTXS.GFPCP (Cruz et al. 1996), flanked by the unique restriction sites AvrII and $\mathrm{XhoI}$ in the PVX cDNA and containing the GFP-2A-CP cassette, was amplified with 5'-AGATTTTCCTAGGCACG-3' (forward) and 5'-CGACCTCGAGTGACAGC-3' (reverse) primers, and the polymerase chain reaction (PCR) product was T/A cloned into pGEM-T Easy (Promega Corp.). The GFP open reading frame was excised from the GFP-CP cassette as an NheI-HindIII fragment and replaced with CFP or mCherry cDNA sequences after amplification of the respective cDNA with primers 5'-GCACCAGC TAGCATCGATCATGGTGAGCAAGGGCGA-3' (forward) and 5'-CTCCCGCAAGCTTAAGAAGGTCAAAATTTCTAGATCCG GATTACTTGTACAGCTCGT-3' (reverse) and digestion of PCR products with NheI-HindIII. The 3.2-kb fragment containing the different FP was then excised as an AvrII-XhoI fragment and ligated into AvrII-XhoI-treated pART27-35S:: PVX.GFP to generate binary vectors 35S::PVX.CFP-CP and 35S::PVX.mCherry-CP. The binary vector pART27-35S::PVX. GFP had been generated by subcloning the 35S-PVX.GFPNOS fragment from a derivative of pPVX204 (Baulcombe et al. 1995 ) into the binary vector pART27 (Gleave 1992) using the

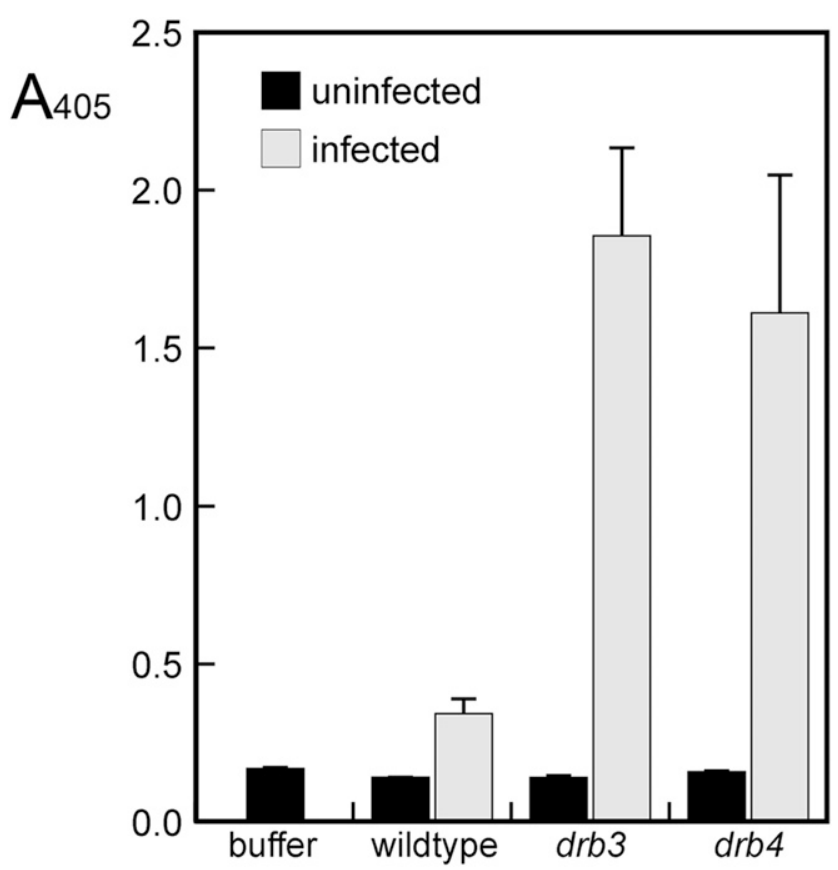

Fig. 7. Loss of double-stranded RNA binding (DRB)3 function gives increased virion accumulation of Turnip mosaic virus (TuMV). Measurement of virion accumulation in TuMV-infected wild type (Col-0), $d r b 3$, and $d r b 4$ Arabidopsis plants by virus-specific enzyme-linked immunosorbent assay 3 weeks after inoculation. The absorbance at $405 \mathrm{~nm}\left(\mathrm{~A}_{405}\right)$ values were measured after a 2-h incubation; $n=5$ infected plants and $n=3$ uninfected plants. Error bars represent the standard error of the mean. 
unique restriction sites NotI and blunted SpeI. A binary expression vector of PVX TGB1 protein N-terminally fused to YFP was generated by PCR amplification of the TGB1 coding sequence from pTXS.GFP-CP (Cruz et al. 1996) with primers 5'-GGATCCCTCGAGATGGATATTCTCATCAGTAG-3' (forward) and 5'-GGATCCGAATTCTGGCCCTGCGCGGACA-TATGTC-3' (reverse). The PCR fragment was then digested with $X h o I$ and EcoRI, and ligated into XhoI-EcoRI-treated pORE1 binary vector (Coutu et al. 2007) harboring 35S::YFP to generate pORE1$35 \mathrm{~S}:: T G B 1-Y F P$. For expression of cytoplasmic GFP and OFP, the respective cDNA were cloned as NcoI-KpnI fragments into pORE1 to give pORE1-35S::GFP and pORE1-35S::OFP. Gateway binary expression vectors driven by the $35 \mathrm{~S}$ promoter for production of N-terminal fusions to a GFP tag (pORE2-GFP Gateway) or mOrange tag (pORE2-OFP Gateway) were constructed as follows. To generate pORE2-GFP Gateway vector, a 4.6-kb cassette from pART7-GFP (Gleave 1992), containing a Cauliflower mosaic virus $35 \mathrm{~S}$ promoter, Gateway recombination sites in frame with GFP cDNA, and the OCS terminator, was excised as a NotI fragment through partial digestion and ligated into pORE2 binary vector (Coutu et al. 2007) linearized with NotI. To make pORE2-OFP Gateway vector, mOrange cDNA was overlap PCR amplified to generate a Sall-ApaI fragment (containing the attR2 site in frame with mOrange and the OCS terminator sequence), which was ligated into pORE2-GFP Gateway digested with SalI and ApaI. The coding sequences of DRB1, DRB2, DRB3, DRB4, and DRB5 of $A$. thaliana and $N$. benthamiana were identified from http://www. arabidopsis.org and http://www.benthgenome.com and PCR amplified using Phusion DNA polymerase (Finnzymes), and cloned into pENTR/D-TOPO (Invitrogen) using primers listed in Supplementary Table S1. The CACC added to forward primers allowed directional cloning into the entry vector and reverse primers eliminated stop codons to allow translational fusion with the FP coding sequence. PCR products captured in $\mathrm{pENTR/D-TOPO}$ were recombined into pORE2-GFP Gateway or pORE2-OFP Gateway vectors for Agrobacterium tumefaciens-mediated plant expression. Functionality of the FP-tagged proteins has not been tested which, in the case of $N$. benthamiana, is explained by the lack of mutants that could be complemented.

\section{Plant material, transformation, and inoculations.}

Transformation of tagged RNAi-protein encoding constructs into Arabidopsis thaliana (Col-0) was by the floral dip method (Clough and Bent 1998). Transformed seed were selected on Murashige and Skoog (MS) agar plates (1\% [wt/vol] sucrose and $0.8 \%$ [wt/vol] agar) supplemented with kanamycin at $50 \mathrm{mg} / \mathrm{liter}$ for 3 weeks. Resistant seedlings were transferred to soil and grown at $22^{\circ} \mathrm{C}$ with a cycle of $16 \mathrm{~h}$ of light and $8 \mathrm{~h}$ of darkness.

A. thaliana plants were grown on MS agar plates for 2 weeks, then transferred to soil and grown for 3 weeks under short-day conditions ( $8 \mathrm{~h}$ of light and $16 \mathrm{~h}$ of darkness) before virus inoculation. Plants were mock inoculated with phosphate buffer or inoculated with sap extracts (diluted in $0.05 \mathrm{M}$ phosphate buffer, pH 7.0) from CMV-, TuMV-, TSWV-, and TYMV-infected plants (viruses were a kind gift from John Randles, University of Adelaide, Australia). Young leaves of transgenic A. thaliana plants showing symptoms of infection were analyzed for expression of the fluorescence-tagged RNAi proteins at 10 to 14 days postinfection. $N$. benthamiana plants were grown in standard greenhouse conditions $\left(23\right.$ to $25^{\circ} \mathrm{C}$, cycle of $16 \mathrm{~h}$ of light and $8 \mathrm{~h}$ of darkness) for 4 weeks before inoculation with sap extracts from CMV-, TuMV-, and TMV-GFP-infected plants. Virus-infected source material for TMV experiments was produced by inoculating $N$. benthamiana with TMV-30B-GFP transcript (Shivprasad et al. 1999), generated using T7 polymerase in vitro transcription from a template of KpnI-linearized plasmid.
The A. thaliana $d r b 3$ and $d r b 4$ T-DNA insertion lines have been previously described (Curtin et al. 2008). Wild-type (Col-0), drb3, and $d r b 4$ seed were planted in soil and grown for 3 weeks in short-day conditions ( $8 \mathrm{~h}$ of light and $16 \mathrm{~h}$ of darkness) at approximately $21^{\circ} \mathrm{C}$. They were mechanically inoculated with TuMV from infected, frozen plant tissue ground in $0.1 \mathrm{M}$ phosphate buffer, $\mathrm{pH}$ 7. Three weeks postinfection, whole plants were harvested and immediately chilled in liquid nitrogen.

\section{ELISA assay.}

TuMV ELISA was performed following the manufacturer's instructions (Agdia). Five biological replicates and two technical replicates were performed for all infected plant lines and treatment conditions, except for the uninfected wild type, for which there were three biological replicates and two technical replicates. Plant tissue was ground in $0.5 \mathrm{ml}$ of extraction buffer $(140 \mathrm{mM}$ $\mathrm{NaCl}, 2 \mathrm{mM} \mathrm{KH} \mathrm{KO}_{4}, 3 \mathrm{mM} \mathrm{Na} \mathrm{HPO}_{4}, 3 \mathrm{mM} \mathrm{KCl}, 300 \mu \mathrm{M}$ $\mathrm{NaN}_{3}, 2 \%$ [wt/vol] polyvinylpyrrolidone, $0.05 \%$ [vol/vol] Tween 20 , and $0.2 \%$ [wt/vol] bovine serum albumin, $\mathrm{pH} 7.4$ ) and chilled for $10 \mathrm{~min}$ on ice before being centrifuged at 3,000 rpm for $30 \mathrm{~min}$ at $4^{\circ} \mathrm{C}$. A 96 -well plate was preprepared with captured antibody and loaded with $100 \mu \mathrm{l}$ of ground sample supernatant. After $2 \mathrm{~h}$, wells were washed with phosphate-buffered saline (PBS) containing $0.05 \%$ (vol/vol) Tween 20. Enzyme conjugate, diluted 1:200 in extraction buffer, was dispensed into wells and incubated for $2 \mathrm{~h}$ before being washed in PBS-Tween. Following this, wells were loaded with phosphatase substrate $(0.6 \mathrm{mg} / \mathrm{ml})$ (Sigma-Aldrich) in PNP buffer $\left(50 \mu \mathrm{M} \mathrm{MgCl}_{2}, 300 \mu \mathrm{M} \mathrm{NaN} \mathrm{N}_{3}\right.$, and $10 \%$ [vol/vol] diethanolamine) for $1 \mathrm{~h}$ at room temperature before absorbance readings were taken at absorbance at $405 \mathrm{~nm}$.

\section{Transient expression in $N$. benthamiana.}

Agrobacterium tumefaciens (strain GV3101) transformed with constructs of interest were grown overnight at $28^{\circ} \mathrm{C}$ in $5 \mathrm{ml}$ of Luria-Bertani medium containing an appropriate selection. After centrifugation, the supernatant was discarded and the bacteria were resuspended in infiltration buffer $\left(10 \mathrm{mM} \mathrm{MgCl}_{2}\right.$ with $150 \mu \mathrm{M}$ acetosyringone and $10 \mathrm{mM} 2$-( $N$-morpholino)ethanesulfonic acid, $\mathrm{pH}$ 5.7) to a concentration of 0.5 at an optical density at $600 \mathrm{~nm}$, except for TGB1-YFP (0.1). After $1 \mathrm{~h}$ of incubation at room temperature, the solution was infiltrated, using a needleless syringe, into leaves of approximately 3 -week-old $N$. benthamiana plants and grown under standard glasshouse conditions $\left(21\right.$ to $23^{\circ} \mathrm{C}, 16 \mathrm{~h}$ of light and $8 \mathrm{~h}$ of darkness). Leaves were imaged 3 to 5 days postinfiltration.

\section{Confocal microscopy.}

Leaf tissue constitutively or transiently expressing FP and either uninfected or infected with a virus was excised from plants and mounted onto a slide in milli-Q water, and the adaxial surface was examined using a Pascal confocal laser-scanning microscope (Carl Zeiss). CFP was excited with the 458-nm laser line of an argon laser and emitted light was captured through a 492- to 508-nm bandpass filter. GFP was excited with a 488-nm argon laser line and emitted light was captured through a 505- to 530-nm bandpass filter. Yellow YFP was excited with a 514-nm argon laser line and emitted light was captured through a 520- to 542-nm bandpass filter. OFP and mCherry were excited with a green $\mathrm{HeNe}$ laser at $543 \mathrm{~nm}$ and emitted light was captured through a 557- to 603-nm bandpass filter. Where GFP expression was low, chlorophyll autofluorescence was also often collected in GFP images. To minimize other potential fluorescence bleed-through, cells containing multiple FP were scanned sequentially, with FP of longer wavelengths scanned before shorter wavelengths. Cells were imaged using a $\times 63$ water-dipping objective lens with a numerical aperture of 0.95, and all images were collected at a single focal plane using LSM 5 software. Figures were constructed using Photoshop CS5 (Adobe Software). 


\section{ACKNOWLEDGMENTS}

We thank K. Chamberlain and K. Shand for assistance in measuring viral accumulation, J. Randles for unrestricted access to his virus collection, S. Santa Cruz for the PVX-overcoat construct, S. Curtin for the primary DRB and AGO clones, and T. Newsome for advice and FP clones. This work was carried out with funding from the Australian Research Council.

\section{LITERATURE CITED}

Baulcombe, D. C., Chapman, S., and Santa Cruz, S. 1995. Jellyfish green fluorescent protein as a reporter for virus infections. Plant J. 7:10451053.

Blevins, T., Rajeswaran, R., Shivaprasad, P. V., Beknazariants, D., SiAmmour, A., Park, H. S., Vazquez, F., Robertson, D., Meins, F., Jr., Hohn, T., and Pooggin, M. M. 2006. Four plant Dicers mediate viral small RNA biogenesis and DNA virus induced silencing. Nucleic Acids Res. 34:6233-6246.

Clough, S. J., and Bent, A. F. 1998. Floral dip: A simplified method for Agrobacterium-mediated transformation of Arabidopsis thaliana. Plant J. 16:735-743.

Cotton, S., Grangeon, R., Thivierge, K., Mathieu, I., Ide, C., Wei, T., Wang, A., and Laliberté, J. F. 2009. Turnip mosaic virus RNA replication complex vesicles are mobile, align with microfilaments, and are each derived from a single viral genome. J. Virol. 83:10460-10471.

Coutu, C., Brandle, J., Brown, D., Brown, K., Miki, B., Simmonds, J., and Hegedus, D. D. 2007. pORE: A modular binary vector series suited for both monocot and dicot plant transformation. Transgenic Res. 16:771-781.

Cruz, S. S., Chapman, S., Roberts, A. G., Roberts, I. M., Prior, D. A., and Oparka, K. J. 1996. Assembly and movement of a plant virus carrying a green fluorescent protein overcoat. Proc. Natl. Acad. Sci. U.S.A. 93: 6286-6290.

Curtin, S. J., Watson, J. M., Smith, N. A., Eamens, A. L., Blanchard, C. L., and Waterhouse, P. M. 2008. The roles of plant dsRNA-binding proteins in RNAi-like pathways. FEBS Lett. 582:2753-2760.

Dalmay, T., Hamilton, A., Rudd, S., Angell, S., and Baulcombe, D. C. 2000 An RNA-dependent RNA polymerase gene in Arabidopsis is required for posttranscriptional gene silencing mediated by a transgene but not by a virus. Cell 101:543-553.

Deleris, A., Gallego-Bartolome, J., Bao, J., Kasschau, K. D., Carrington, J. C., and Voinnet, O. 2006. Hierarchical action and inhibition of plant Dicer-like proteins in antiviral defense. Science 313:68-71.

Ding, S. W. 2010. RNA-based antiviral immunity. Nat. Rev. Immunol. 10: 632-644.

Ding, S. W., and Voinnet, O. 2007. Antiviral immunity directed by small RNAs. Cell 130:413-426.

Eamens, A., Wang, M. B., Smith, N. A., and Waterhouse, P. M. 2008. RNA silencing in plants: Yesterday, today, and tomorrow. Plant Physiol. 147: 456-468.

Eamens, A. L., Wook Kim, K., and Waterhouse, P. M. 2012. DRB2, DRB3 and DRB5 function in a non-canonical microRNA pathway in Arabidopsis thaliana. Plant Signal. Behav. 7:1224-1229.

Fusaro, A. F., Matthew, L., Smith, N. A., Curtin, S. J., Dedic-Hagan, J., Ellacott, G. A., Watson, J. M., Wang, M. B., Brosnan, C., Carroll, B. J., and Waterhouse, P. M. 2006. RNA interference-inducing hairpin RNAs in plants act through the viral defence pathway. EMBO Rep. 7:1168-1175.

Garcia-Ruiz, H., Carbonell, A., Hoyer, J. S., Fahlgren, N., Gilbert, K. B., Takeda, A., Giampetruzzi, A., Garcia Ruiz, M. T., McGinn, M. G., Lowery, N., Martinez Baladejo, M. T., and Carrington, J. C. 2015. Roles and programming of Arabidopsis ARGONAUTE proteins during Turnip mosaic virus infection. PLoS Pathog. 11:e1004755.

Gleave, A. P. 1992. A versatile binary vector system with a T-DNA organisational structure conducive to efficient integration of cloned DNA into the plant genome. Plant Mol. Biol. 20:1203-1207.

Hamilton, A. J., and Baulcombe, D. C. 1999. A species of small antisense RNA in posttranscriptional gene silencing in plants. Science 286: 950-952.

Hiraguri, A., Itoh, R., Kondo, N., Nomura, Y., Aizawa, D., Murai, Y., Koiwa, H., Seki, M., Shinozaki, K., and Fukuhara, T. 2005. Specific interactions between Dicer-like proteins and HYL1/DRB-family dsRNA-binding proteins in Arabidopsis thaliana. Plant Mol. Biol. 57:173-188.

Hoffer, P., Ivashuta, S., Pontes, O., Vitins, A., Pikaard, C., Mroczka, A., Wagner, N., and Voelker, T. 2011. Posttranscriptional gene silencing in nuclei. Proc. Natl. Acad. Sci. U.S.A. 108:409-414.

Jakubiec, A., Yang, S. W., and Chua, N.-H. 2012. Arabidopsis DRB4 protein in antiviral defense against Turnip yellow mosaic virus infection. Plant J. 69:14-25.
King, A. M. Q., Adams, M. J., Carstens, E. B., and Lefkowitz, E. J., eds. 2011. Virus Taxonomy: Classification and Nomenclature of Viruses, The Ninth Report of the ICTV. Online publication. International Committee on Taxonomy of Viruses. https://talk.ictvonline.org/ictv-reports/ictv_9th_report/

Lindbo, J. A., and Dougherty, W. G. 1992. Pathogen-derived resistance to a potyvirus: Immune and resistant phenotypes in transgenic tobacco expressing altered forms of a potyvirus coat protein nucleotide sequence. Mol. Plant-Microbe Interact. 5:144-153.

Linnik, O., Liesche, J., Tilsner, J., and Oparka, K. J. 2013. Unraveling the structure of viral replication complexes at super-resolution. Front. Plant Sci. 4:6.

Llave, C. 2010. Virus-derived small interfering RNAs at the core of plantvirus interactions. Trends Plant Sci. 15:701-707.

Lucas, W. J. 2006. Plant viral movement proteins: Agents for cell-to-cell trafficking of viral genomes. Virology 344:169-184.

Mandadi, K. K., and Scholthof, K. B. 2013. Plant immune responses against viruses: How does a virus cause disease? Plant Cell 25:1489-1505.

Margis, R., Fusaro, A. F., Smith, N. A., Curtin, S. J., Watson, J. M., Finnegan, E. J., and Waterhouse, P. M. 2006. The evolution and diversification of Dicers in plants. FEBS Lett. 580:2442-2450.

Mlotshwa, S., Pruss, G. J., and Vance, V. 2008. Small RNAs in viral infection and host defense. Trends Plant Sci. 13:375-382.

Mourrain, P., Béclin, C., Elmayan, T., Feuerbach, F., Godon, C., Morel, J. B., Jouette, D., Lacombe, A. M., Nikic, S., Picault, N., Rémoué, K., Sanial, M., Vo, T. A., and Vaucheret, H. 2000. Arabidopsis SGS2 and SGS3 genes are required for posttranscriptional gene silencing and natural virus resistance. Cell 101:533-542.

Pontes, O., Vitins, A., Ream, T. S., Hong, E., Pikaard, C. S., and CostaNunes, P. 2013. Intersection of small RNA pathways in Arabidopsis thaliana sub-nuclear domains. PLoS One 8:e65652.

Pumplin, N., Sarazin, A., Jullien, P. E., Bologna, N. G., Oberlin, S., and Voinnet, O. 2016. DNA methylation influences the expression of DICER-LIKE 4 isoforms, which encode proteins of alternative localization and function. Plant Cell 28:2786-2804; advance online publication.

Qu, F., Ye, X., and Morris, T. J. 2008. Arabidopsis DRB4, AGO1, AGO7, and RDR6 participate in a DCL4-initiated antiviral RNA silencing pathway negatively regulated by DCL1. Proc. Natl. Acad. Sci. U.S.A. 105:14732-14737.

Raja, P., Jackel, J. N., Li, S., Heard, I. M., and Bisaro, D. M. 2014 Arabidopsis double-stranded RNA binding protein DRB3 participates in methylation-mediated defense against geminiviruses. J. Virol. 88:2611-2622.

Reis, R. S., Hart-Smith, G., Eamens, A. L., Wilkins, M. R., and Waterhouse, P. M. 2015. Gene regulation by translational inhibition is determined by Dicer partnering proteins. Nat. Plants 1:Article 14027. doi:10.1038/nplants. 2014.27

Schoelz, J. E., Harries, P. A., and Nelson, R. S. 2011. Intracellular transport of plant viruses: Finding the door out of the cell. Mol. Plant 4:813-831.

Shivprasad, S., Pogue, G. P., Lewandowski, D. J., Hidalgo, J., Donson, J. Grill, L. K., and Dawson, W. O. 1999. Heterologous sequences greatly affect foreign gene expression in tobacco mosaic virus-based vectors. Virology 255:312-323.

Tilsner, J., Linnik, O., Christensen, N. M., Bell, K., Roberts, I. M., Lacomme, C., and Oparka, K. J. 2009. Live-cell imaging of viral RNA genomes using a Pumilio-based reporter. Plant J. 57:758-770.

Tilsner, J., Linnik, O., Louveaux, M., Roberts, I. M., Chapman, S. N., and Oparka, K. J. 2013. Replication and trafficking of a plant virus are coupled at the entrances of plasmodesmata. J. Cell Biol. 201:981-995.

Tilsner, J., Linnik, O., Wright, K. M., Bell, K., Roberts, A. G., Lacomme, C., Santa Cruz, S., and Oparka, K. J. 2012. The TGB1 movement protein of Potato virus $X$ reorganizes actin and endomembranes into the $\mathrm{X}$-body, a viral replication factory. Plant Physiol. 158:1359-1370.

Vaucheret, H. 2008. Plant ARGONAUTES. Trends Plant Sci. 13:350-358

Verchot-Lubicz, J., Torrance, L., Solovyev, A. G., Morozov, S. Y., Jackson, A. O., and Gilmer, D. 2010. Varied movement strategies employed by triple gene block-encoding viruses. Mol. Plant-Microbe Interact. 23 1231-1247.

Voinnet, O., Pinto, Y. M., and Baulcombe, D. C. 1999. Suppression of gene silencing: A general strategy used by diverse DNA and RNA viruses of plants. Proc. Natl. Acad. Sci. U.S.A. 96:14147-14152.

Wang, M. B., Abbott, D. C., and Waterhouse, P. M. 2000. A single copy of a virus-derived transgene encoding hairpin RNA gives immunity to barley yellow dwarf virus. Mol. Plant Pathol. 1:347-356.

Waterhouse, P. M., and Fusaro, A. F. 2006. Plant science. Viruses face a double defense by plant small RNAs. Science 313:54-55.

Waterhouse, P. M., Graham, M. W., and Wang, M. B. 1998. Virus resistance and gene silencing in plants can be induced by simultaneous expression of sense and antisense RNA. Proc. Natl. Acad. Sci. U.S.A. 95: 13959-13964. 